Original article

\title{
Association between prior somatic disease and 5-year relapse risk among 11,856 incident patients with schizophrenia
}

\author{
Ole Köhler-Forsberga,b,c,d,*, Holger J. Sørensen ${ }^{\mathrm{d}}$, Michael E. Benros ${ }^{\mathrm{d}}$, \\ Liselotte Petersen $^{\mathrm{c}, \mathrm{e}, \mathrm{f}}$, Christiane Gasse ${ }^{\mathrm{a}, \mathrm{c}, \mathrm{f}, \mathrm{g}}$ \\ a Psychosis Research Unit, Aarhus University Hospital Psychiatry, Aarhus, Denmark \\ b Department of Clinical Medicine, Aarhus University Hospital, Aarhus, Denmark \\ ${ }^{\mathrm{c}}$ National Centre for Register-Based Research, Aarhus University, Denmark \\ d Mental Health Centre Copenhagen, Faculty of Health Sciences, University of Copenhagen, Copenhagen, Denmark \\ e Centre for Integrated Register-Based Research (CIRRAU), Aarhus University, Aarhus, Denmark \\ ${ }^{\mathrm{f}}$ The Lundbeck Foundation Initiative for Integrative Psychiatric Research, iPSYCH, Denmark \\ ${ }^{g}$ Department for Depression and Anxiety, Aarhus University Hospital Psychiatry, Aarhus, Denmark
}

\section{A R T I C L E I N F O}

\section{Article history:}

Received 29 January 2019

Received in revised form 27 March 2019

Accepted 30 March 2019

Available online 10 April 2019

\section{Keywords:}

Schizophrenia

Somatic comorbidity

Relapse

Early intervention

Early onset schizophrenia

Somatic diseases

Epidemiology

Register-based

\begin{abstract}
A B S T R A C T
Background: Somatic diseases have been associated with an increased risk for subsequent schizophrenia; however, it is unknown whether prior somatic diseases negatively affect early treatment outcomes after a first-time schizophrenia diagnosis.

Methods: We included all individuals born in Denmark after January $1^{\text {st }}, 1977$ and first-time diagnosed with schizophrenia between January $1^{\text {st }}, 1996$ and December $31^{\text {st }}, 2015$. We identified all life-time somatic hospital contacts and all prescriptions within the year before the first-time schizophrenia diagnosis and followed patients for up to five years regarding risk for schizophrenia (re)-hospitalization (relapse). We performed Cox regression analyses calculating hazard rate ratios (HRR) including 95\%confidence intervals (CI) and adjusted for relevant confounders.

Results: We followed a total of 11,856 patients with a first-time schizophrenia diagnosis ( $58.7 \%$ male, mean age $23.1(\mathrm{SD}=4.7)$ years) for 39,033 person-years, whereof $5506(46.4 \%)$ had relapse with schizophrenia re-hospitalization during 5-year of follow-up. Somatic hospital contacts ever before (95.4\%; HRR $=1.30 ; 95 \%-\mathrm{CI}=1.07-1.59)$, and specifically during the year before schizophrenia diagnosis $(42.5 \% ; \mathrm{HRR}=1.36 ; 95 \%-\mathrm{CI}=1.11-1.66)$ were associated with an increased risk of schizophrenia relapse as were a greater number of prior somatic hospital contacts $(p<0.001)$. Individuals with up to four different prescriptions for somatic medications showed a trend towards a slightly lower risk of relapse. Conclusion: Somatic diseases and health seeking patterns might have an impact on the course of schizophrenia, where severe somatic comorbidity, specifically during the year before first-time schizophrenia diagnosis, seem to negatively affect early treatment course, whereas previous somatic medication use may indicate a better compliance and help-seeking behavior.
\end{abstract}

(c) 2019 Elsevier Masson SAS. All rights reserved.

\section{Background}

The vast majority (96\%) of patients with a first-time schizophrenia diagnosis had previously been diagnosed with somatic diseases and a broad range of somatic diseases are associated with an increased risk of schizophrenia [1]. Particularly epilepsy [1], brain injury [1,2], infections [3-6] autoimmune diseases [3], and asthma [7] have been associated with increased risk of schizophrenia.

\footnotetext{
* Corresponding author at: Psychosis Research Unit, Aarhus University Hospital Psychiatry, Palle Juul-Jensens Boulevard 175, 8200 Aarhus N, Denmark.

E-mail address: karkoe@rm.dk (O. Köhler-Forsberg).
}

However, it is important to extend these findings beyond an etiological perspective and investigate whether somatic conditions affect the early clinical course and readmission risk after a schizophrenia diagnosis. Only a few smaller clinical studies among patients with mental disorders have indicated that somatic conditions may result in a longer duration of illness and an increased readmission risk [8,9], thus indicating that somatic diseases may affect the clinical course of the mental disorder. Nevertheless, these studies were limited by small study populations and by not including schizophrenia specifically or specific somatic diseases nor the timing of the somatic diseases, i.e. whether the somatic diseases occurred before or after the mental disorder diagnosis. Furthermore, it is important to investigate the 
early clinical course among patients with a first-time schizophrenia diagnosis since this time-frame has been emphasized as highly important for long-term treatment outcomes [10,11].

Hence, we hypothesized that somatic diseases diagnosed prior to the incident schizophrenia diagnosis would be associated with an increased risk for schizophrenia relapse during the first five years after the first-time diagnosis. Our associated hypothesis was that the specific somatic diseases that have been associated with subsequent development of schizophrenia as described above ${ }^{1-7}$ also would be associated with an increased risk for schizophrenia re-hospitalizations.

\section{Methods}

The present study is a register-based cohort study based on the Danish nationwide registers [12-16]. The unique personal identification number enables individual linkage between all registers. This study has been approved by the Danish Data Protection Agency, the Danish National Board of Health and Statistics Denmark (Danish Data Protection Agency: Journal number: 2015-57-0002 / Journal number: 62908 (Joint Registration for Aarhus University); National Board of Health registration number: FSEID 00000098).

\subsection{Study population}

The Danish Psychiatric Central Research Register has covered all mental disorders diagnosed within the secondary healthcare system since 1969 [12] and the Danish National Patient Registry has covered all somatic diseases diagnosed in the secondary healthcare system since 1977 [13]. Both registers have included all outpatient contacts for mental disorders and somatic diseases, respectively, since 1995 . We conducted a nationwide cohort study covering all individuals born in Denmark after January $1^{\text {st }}, 1977$ [14] to be able to assess life-time somatic diseases and mental disorders. We identified individuals with a first-time schizophrenia diagnosis (International Classification of Diseases, $8^{\text {th }}$ edition (ICD-8): 295. $\times 9$ excluding 295.79; ICD-10: F20.X) between January, $1^{\text {st }} 1996$ and December $31^{\text {st }}, 2015$ during admission or an outpatient setting (age $\geq 10$ years) [12]. A total of 12 individuals were excluded due to a diagnosis before the age of 10 . We included only individuals diagnosed with a first-time schizophrenia diagnosis after January $1^{\text {st }}, 1996$, since the Danish National Prescription Registry [15] contains nationwide information on all redeemed prescriptions after January $1^{\text {st }}, 1995$, allowing inclusion of previous somatic medication use and adjustment for previous psychotropic drug use. The validity of the schizophrenia diagnosis in the registers is high with $97.5 \%$ of patients with a registered schizophrenia diagnosis fulfilling ICD-10 criteria [17].

\subsection{Follow-up}

The index date was the day of first schizophrenia diagnosis. Among patients diagnosed during an admission, this was the discharge date, and among outpatients this was the day of initiation of the outpatient contact. We followed all individuals from the index date for a maximum of five years until first outcome (see below), death, emigration, or end of the study period on April $10^{\text {th }}, 2017$.

\subsection{Outcome - schizophrenia relapse}

We identified the first inpatient hospitalization with schizophrenia (ICD-10: F20.X) between January $15^{\text {th }}, 1996$ and April $10^{\text {th }}$, 2017 during the first five years after the incident schizophrenia diagnosis via the Danish Psychiatric Central Research Register [12].
Hospitalizations for schizophrenia have been evaluated as an adequate proxy for relapse in schizophrenia [18]. The hospitalization had to occur at least 14 days after the first-time schizophrenia diagnosis. Thereby, we avoided hospitalizations shortly after or in connection with initiation of an outpatient contact, and discharges lasting only few days.

\subsection{Exposure - somatic diseases}

To cover all treated somatic diseases, we identified 1) any lifetime somatic disease diagnosed in a hospital setting before the first-time schizophrenia diagnosis and 2) any somatic medication use during the year before the first-time schizophrenia diagnosis.

Via the Danish National Patient Registry [13], we identified all somatic hospital contacts since birth and prior to first-time schizophrenia diagnosis (ICD-codes supplied in Supplementary Table 1). We included the main diagnoses from hospitalizations, outpatient contacts and emergency room contacts. In addition to the main ICD-categories, we were interested in specific disease entities, which have been associated with an increased risk of subsequent schizophrenia diagnosis: Epilepsy [1], brain injury [2], autoimmune diseases [3], and asthma including other atopic diseases [7] (Supplementary Table 1). To evaluate the influence of chronic diseases, we identified diagnoses included in the Charlson comorbidity score [19], covering 19 severe chronic conditions (myocardial infarct, congestive heart failure, peripheral vascular disease, cerebrovascular disease, dementia, chronic pulmonary disease, connective tissue disease, ulcer disease, mild liver disease, diabetes, hemiplegia, moderate to severe renal disease, diabetes with end organ damage, any tumor, leukemia, lymphoma, moderate to severe liver disease, metastatic solid tumor, AIDS). These diagnoses were identified from main diagnoses at in- or outpatient contacts to somatic hospitals only. The Charlson score has been validated [20] and modified for use with the ICD-10 [21].

We excluded individuals who had only received the following diagnostic codes: Symptoms, signs and abnormal clinical and laboratory findings, not elsewhere classified (ICD-8: 780, 782.3, 782.5-791, 793-796; ICD-10: R00-R99), and factors influencing health status and contact with health services (ICD-10: Z00-Z99).

In addition to hospital contacts and as a proxy for contacts with the general practitioner, we used the Danish National Prescription Registry [15] to identify all redeemed prescriptions for somatic medications (i.e., except for psychotropic drugs defined as Anatomical Therapeutic Chemical (ATC) codes: N03N07) within the year before the first-time schizophrenia diagnosis, as prescriptions indicate somatic diseases treated in the primary sector (ATC-codes supplied in Supplementary Table 2). We calculated the amount of prescriptions from different ATC groups.

\subsection{Assessment of covariates}

We assessed sociodemographic covariates at the date of firsttime schizophrenia diagnosis: age, sex [14], and educational level (divided into primary school and higher education) [16]. Regarding the severity of schizophrenia, we identified whether individuals were in- or outpatients at first-time schizophrenia diagnosis including the length of hospitalization. In addition, the following psychiatric diagnoses prior to incident schizophrenia diagnosis were identified [12]: Disorders due to use of alcohol or other psychoactive substances, bipolar disorder, depression, and anxiety disorders (codes supplied in Supplementary Table 3). Via the Danish National Prescription Registry [15], we included information on any use of psychotropic medication (ATC-code: N03-N07) and specifically use of antipsychotics (N05A), anxiolytics (N05B), hypnotics (N05C) and antidepressants (N06A) within the year prior 
to first-time schizophrenia diagnosis, since prior treatment may influence both treatment course and the risk of somatic diseases by induction of metabolic disturbances. Furthermore, we identified information on any parental psychiatric diagnosis since 1969 (ICD8: 290-315; ICD-10: F00-99) [12] and the highest educational level achieved by the parents ${ }^{16}$ at the time of first-time schizophrenia diagnosis.

\subsection{Statistical analyses}

First, to evaluate differences in baseline characteristics between people with and without previous somatic hospital contacts and somatic medication use, we performed logistic regression analyses and report odds ratios (OR) including 95\%-confidence intervals (95\%-CI). In addition, we calculated the annual prevalence of individuals with a somatic hospital contact during the 10 years prior to the first-time schizophrenia diagnosis.

Second, concerning the association between previous somatic hospital contacts or somatic medication use and hospitalizations with schizophrenia during the subsequent five years, we performed Cox regression analyses and report hazard rate ratios (HRR) and 95\%-CI. We studied the association between 1 ) any lifetime somatic hospital contact and 2) any somatic medication use during the year before the first-time schizophrenia diagnosis with the risk of subsequent re-hospitalization with schizophrenia during the first five years after the first-time schizophrenia diagnosis. Furthermore, we performed analyses that included the different categories of somatic hospital contacts and specific somatic disease categories. The latter analyses were adjusted for multiple testing by dividing the p-value of 0.05 with the number of tests performed.

The adjusted analyses were adjusted for age, gender, and year of diagnosis. The fully adjusted analyses were adjusted for age, gender, year of diagnosis, in- or outpatient status at first-time schizophrenia diagnosis, length of index hospitalization, psychiatric diagnoses prior to first-time schizophrenia diagnosis, highest educational level, use of psychotropics within the year prior to first-time diagnosis, parental psychiatric diagnoses and highest parental educational level. All analyses were mutually adjusted, i.e. analyses on somatic hospital contacts were adjusted for previous somatic medication use and vice versa.

Furthermore, we investigated the amount of different prior somatic diseases, which included analyses on the amount of somatic hospital contacts with different somatic diseases and the amount of different somatic medication groups. In addition, since some somatic diseases may have occurred several years before the first schizophrenia diagnosis, and thus not may be relevant for the course of the schizophrenia disorder, we investigated the association between the time since the last somatic hospital contact prior to first-time schizophrenia diagnosis. To investigate on possible linear relationships, we performed Wald's test and report p-values.

\subsection{Post-hoc analysis}

First, having observed that somatic hospital contacts during the year before the first-time schizophrenia diagnosis were associated with a significantly increased risk of subsequent hospitalization with schizophrenia, we performed multiplicative interaction analyses between somatic hospital contacts during the year before the first-time schizophrenia diagnosis and somatic medication use during the year before the first-time schizophrenia diagnosis. Second, we analyzed the association between the amount of different somatic hospital contacts during the year before the firsttime schizophrenia diagnosis and risk of schizophrenia rehospitalization. Third, since we found large gender differences regarding baseline characteristics (Table 1), we performed gender interaction analyses.

\section{Results}

Among 11,856 individuals diagnosed with schizophrenia between 1996 and 2015 (follow-up of 39,033.3 person-years), a total of $11,312(95.4 \%)$ had been hospitalized in a somatic hospital prior to first-time schizophrenia diagnosis and 7243 (61.1\%) had redeemed at least one prescription for somatic medications during the previous year. We identified the following baseline characteristics to be associated with somatic hospital contacts prior to firsttime schizophrenia diagnosis (Table 1): a later index year, a prior diagnosis with substance abuse, and a maternal mental disorder (all $\mathrm{p}<0.05$ ). Somatic medication use during the previous year was associated with female gender, a later index year, and previous psychotropic drug use (all $\mathrm{p}<0.05)$. Fig. 1 shows an increase of individuals with a contact to a somatic hospital during the 10 years prior to the first-time schizophrenia diagnosis.

\subsection{Prior somatic hospital contacts and risk of relapse}

Among the 11,856 individuals diagnosed with schizophrenia, 5506 (46.4\%) were re-hospitalized with schizophrenia during the first five years of follow-up. The occurrence of any somatic hospital contact at any time prior to first-time schizophrenia diagnosis was associated with an increased risk of hospitalization with schizophrenia by a HRR of $1.30(95 \%-\mathrm{CI}=1.07 ; 1.59)$ during the first five years (Table 2 ).

Several specific somatic diseases and diagnostic categories were associated with an increased risk for re-hospitalization with schizophrenia, which remained significant after adjustment for multiple testing (Table 2). Concerning chronic diseases, individuals with diagnoses included in the Charlson comorbidity score were associated with an increased risk for re-hospitalization with schizophrenia. However, we found no association between a Charlson comorbidity score of three or more and re-hospitalization ( $\mathrm{HR}=0.79 ; 95 \%-\mathrm{Cl}=0.54-1.15)$.

Table 3 shows a relationship ( $\mathrm{p}<0.005$ ) between the number of somatic hospital contacts prior to first-time schizophrenia diagnosis and the risk for hospitalization with schizophrenia, with 6 or more prior somatic hospital contacts increasing the risk of hospitalization with schizophrenia by a HR of 1.61 (95\%-CI=1.29; 2.00$)$.

When studying the temporal associations (Table 3 ), we found that it was only somatic hospital contacts within the year prior to the first-time schizophrenia diagnosis that were associated with an increased HRR of $1.36(95 \%-\mathrm{CI}=1.11 ; 1.66)$ for re-hospitalization with schizophrenia during the subsequent five years.

\subsection{Prior somatic medication use and risk of relapse}

When investigating individuals who had redeemed a prescription for any somatic medication during the year prior to first-time schizophrenia diagnosis we observed an inverse relationship, where redeemed prescriptions for any somatic medication during the year prior to first-time schizophrenia diagnosis were associated with a non-significantly slightly decreased risk for 5year-(re) hospitalization with schizophrenia (HRR of 0.96; 95\%$\mathrm{CI}=0.90 ; 1.02$ ) (Table 2 ). The risk estimates were significantly decreased among individuals who redeemed two or four different prescriptions (Fig. 2 \& Supplementary Table 4).

\subsection{Post-hoc analysis}

First, interaction analyses between somatic hospital contacts and somatic medication use within the year before the first-time 
Table 1

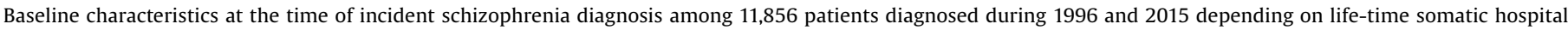
contacts and somatic medication use during the year before the schizophrenia diagnosis.

\begin{tabular}{|c|c|c|c|c|c|}
\hline & Total & $\begin{array}{l}\text { Any somatic hospital } \\
\text { contact }\end{array}$ & $\begin{array}{l}\text { Any vs. no somatic hospital contact, } \\
\text { OR }^{\mathrm{a}}(95 \%-\mathrm{CI})\end{array}$ & $\begin{array}{l}\text { Any somatic } \\
\text { medication }\end{array}$ & $\begin{array}{l}\text { Any vs. no somatic medication, } \mathrm{OR}^{\mathrm{a}} \\
(95 \%-\mathrm{CI})\end{array}$ \\
\hline $\mathrm{N}(\%)$ & $11,856(100)$ & $11,312(95.4)$ & & $7,243(61.1)$ & \\
\hline \multicolumn{6}{|l|}{ Gender } \\
\hline Male & $6,960(58.7)$ & $6,599(58.3)$ & 1.0 (ref.) & $3,313(45.7)$ & 1.0 (ref.) \\
\hline Female & $4,896(41.3)$ & $4,713(41.7)$ & $1.22(0.93 ; 1.61)$ & $3,930(54.3)$ & $4.28(3.87 ; 4.72)$ \\
\hline Mean age, years (IQR) & $\begin{array}{l}23.1(19.6 \\
25.8)\end{array}$ & $23.1(19.6 ; 25.8)$ & $1.0(0.97 ; 1.04)$ & $23.0(19.5 ; 25.7)$ & \\
\hline \multicolumn{6}{|l|}{ Age group (years): } \\
\hline$<18$ & $1,360(11.5)$ & $1,287(11.4)$ & 1.0 (ref.) & $836(11.5)$ & 1.0 (ref.) \\
\hline $18-<22$ & $4,239(35.7)$ & $4,057(35.8)$ & $1.07(0.73 ; 1.57)$ & $2,675(36.9)$ & $1.00(0.86 ; 1.16)$ \\
\hline $22-<25$ & $2,722(23.0)$ & $2,591(22.9)$ & $1.06(0.70 ; 1.61)$ & $1,634(22.6)$ & $0.94(0.80 ; 1.10)$ \\
\hline $25-<28$ & $1,642(13.9)$ & $1,576(14.0)$ & $1.34(0.78 ; 2.30)$ & $949(13.1)$ & $0.90(76 ; 1.08)$ \\
\hline$\geq 28$ & $1,893(16.0)$ & $1,801(16.0)$ & $1.03(0.58 ; 1.82)$ & $1,149(16.0)$ & $0.93(0.78 ; 1.11)$ \\
\hline \multicolumn{6}{|l|}{ Index year } \\
\hline 1996-2003 & $1,815(15.3)$ & $1,646(14.6)$ & 1.0 (ref.) & $1,010(13.9)$ & 1.0 (ref.) \\
\hline 2004-2009 & $3,665(30.9)$ & $3,480(30.8)$ & $2.42(1.78 ; 3.28)$ & $2,234(30.8)$ & $1.10(0.96 ; 1.25)$ \\
\hline 2010-2015 & $6,376(53.8)$ & $6,186(54.7)$ & $6.89(4.81 ; 9.86)$ & $3,999(55.2)$ & $1.15(1.01 ; 1.31)$ \\
\hline \multicolumn{6}{|l|}{ Patient-type } \\
\hline Inpatient & 3,797 (32.0) & 3,394 (29.9) & 1.0 (ref.) & $2,194(30.3)$ & 1.0 (ref.) \\
\hline Outpatient & $8,059(68.0)$ & $7,941(70.1)$ & $1.02(0.87 ; 1.19)$ & $5,049(69.7)$ & $1.02(0.97 ; 1.08)$ \\
\hline $\begin{array}{l}\text { Mean days (SD) baseline } \\
\text { hospitalization }\end{array}$ & $75.1(111.3)$ & $75.6(113.0)$ & $1.0(0.99 ; 1.01)$ & $67.4(102.9)$ & $1.00(0.99 ; 1.00)$ \\
\hline \multicolumn{6}{|l|}{ Education } \\
\hline Primary school & 2,989 (25.3) & $2,843(25.4)$ & 1.0 (ref.) & $1,814(25.2)$ & 1.0 (ref.) \\
\hline Higher education & $8,867(74.7)$ & $8,469(74.6)$ & $0.90(0.67 ; 1.21)$ & $5,429(74.8)$ & $0.96(0.87 ; 1.06)$ \\
\hline \multicolumn{6}{|l|}{ Prior psychiatric diagnoses } \\
\hline Substance abuse & $2,061(17.4)$ & $2,010(17.8)$ & $2.48(1.53 ; 4.02)$ & $1,139(15.7)$ & $0.97(0.87 ; 1.09)$ \\
\hline Depression & $2,548(21.5)$ & $2,494(22.0)$ & $1.29(0.90 ; 1.86)$ & $1,817(25.1)$ & $1.10(0.98 ; 1.24)$ \\
\hline Bipolar disorder & $261(2.2)$ & $253(2.2)$ & $1.18(0.35 ; 4.01)$ & $170(2.4)$ & $0.72(0.52 ; 1.01)$ \\
\hline Anxiety & $1,332(11.2)$ & $1,299(11.5)$ & $1.06(0.64 ; 1.74)$ & $941(13.0)$ & $1.07(0.91 ; 1.25)$ \\
\hline \multicolumn{6}{|l|}{ Prior psychotropic drug use ${ }^{b}$} \\
\hline No use & $4,427(37.3)$ & $4,249(37.5)$ & 1.0 (ref.) & $2,075(28.7)$ & 1.0 (ref.) \\
\hline Any use & $7,429(62.7)$ & $7,087(62.5)$ & $1.17(0.75 ; 1.82)$ & $5,168(71.4)$ & $1.39(1.20 ; 1.60)$ \\
\hline Antipsychotics & $5,128(43.3)$ & $4,968(43.9)$ & $1.39(0.97 ; 1.99)$ & $3,578(49.4)$ & $1.18(1.04 ; 1.32)$ \\
\hline Anxiolytics & $1,257(10.3)$ & $1,219(10.8)$ & $1.23(0.76 ; 2.00)$ & $963(13.3)$ & $1.51(1.29 ; 1.78)$ \\
\hline Hypnotics & $1,446(12.2)$ & $1,412(12.5)$ & $1.32(0.81 ; 2.13)$ & $1,090(15.1)$ & $1.20(1.04 ; 1.39)$ \\
\hline Antidepressants & $4,466(37.7)$ & $4,336(38.3)$ & $0.80(0.54 ; 1.1 .18)$ & $3,329(46.0)$ & $1.46(1.30 ; 1.64)$ \\
\hline \multicolumn{6}{|c|}{ Parental psychiatric diagnoses } \\
\hline No diagnosis & $8,922(75.2)$ & $8,444(74.5)$ & 1.0 (ref.) & $5,413(74.6)$ & 1.0 (ref.) \\
\hline Diagnosis of the mother & $1,913(16.5)$ & $1,917(16.9)$ & $2.04(1.28 ; 3.26)$ & $1,232(17.0)$ & $1.08(0.96 ; 1.21)$ \\
\hline Diagnosis of the father & $1,339(11.5)$ & $1,341(11.8)$ & $1.38(0.85 ; 2.26)$ & $845(11.7)$ & $0.96(0.84 ; 1.10)$ \\
\hline \multicolumn{6}{|l|}{ Highest parental education } \\
\hline Primary school & $3,040(28.1)$ & $2,959(27.9)$ & 1.0 (ref.) & $1,799(24.8)$ & 1.0 (ref.) \\
\hline Higher education & $7,806(71.9)$ & $7,639(72.1)$ & $1.26(0.96 ; 1.66)$ & $4,984(68.8)$ & $1.01(0.92 ; 1.12)$ \\
\hline
\end{tabular}

Abbreviations: OR=Odds Ratio; 95\%-CI=95\%-Confidence Interval. Bold values represent significant results.

a The ORs are based on logistic regression analyses and all results are adjusted for all covariates mentioned in Table 1.

b Within the year prior to first schizophrenia diagnosis.

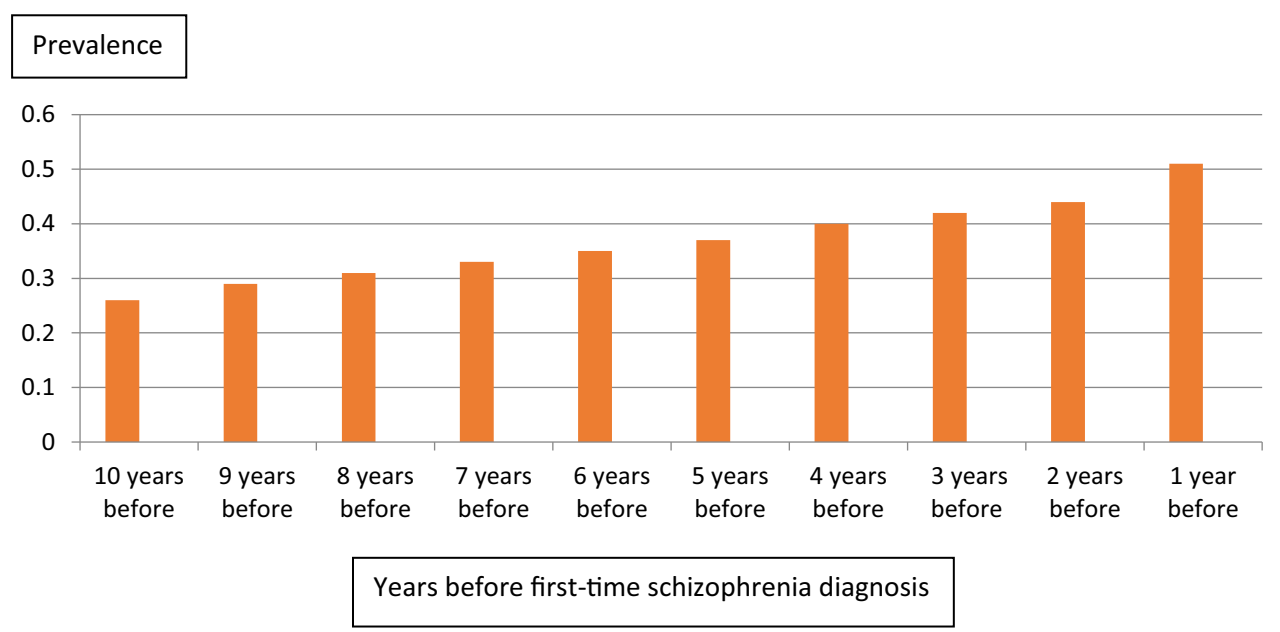

Fig. 1. Annual prevalence of individuals with a contact to a somatic hospital during the 10 years prior to first-time schizophrenia diagnosis among 11,856 patients. 
Table 2

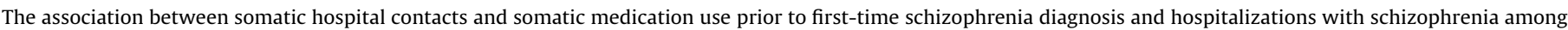
11,856 incident patients with schizophrenia.

\begin{tabular}{|c|c|c|c|c|}
\hline & \multirow{2}{*}{$\begin{array}{l}\text { Total } \\
\text { N (\%) }\end{array}$} & \multicolumn{3}{|c|}{ Re-Hospitalization within 5 years } \\
\hline & & $\mathrm{N}(\%)$ & Crude HRR $^{\mathrm{a}}(95 \%-\mathrm{CI})$ & Adjusted $\mathrm{HRR}^{\mathrm{b}}(95 \%-\mathrm{CI})$ \\
\hline Individuals with schizophrenia & $11,856(100)$ & $5,506(46.4)$ & & \\
\hline No somatic hospital (ref) & $544(4.6)$ & $220(40.4)$ & 1.00 (ref.) & 1.00 (ref.) \\
\hline Any somatic hospital contact & $11,312(95.4)$ & $5,286(46.7)$ & $1.22(1.06 ; 1.39)$ & $1.30(1.07 ; 1.59)$ \\
\hline No somatic medication during the previous year ${ }^{\mathrm{e}}$ (ref) & $4,613(38.9)$ & $2,155(46.7)$ & 1.00 (ref.) & 1.00 (ref.) \\
\hline Any somatic medication during the previous year ${ }^{e}$ & $7,243(61.1)$ & $3,351(46.3)$ & $0.92(0.88 ; 0.98)$ & $0.96(0.90 ; 1.02)$ \\
\hline \multicolumn{5}{|l|}{ The specific disease categories resulting in somatic hospital contacts } \\
\hline Infectious and parasitic diseases ${ }^{\mathrm{c}}$ & $1,911(16.1)$ & $955(50.0)$ & $1.41(1.22 ; 1.64)$ & $1.42(1.16 ; 1.75)$ \\
\hline Neoplasms ${ }^{\mathrm{c}}$ & $453(3.8)$ & $226(49.9)$ & $1.38(1.15 ; 1.66)$ & $1.46(1.16 ; 1.85)$ \\
\hline Diseases of the blood (forming) organs, immunological disorders ${ }^{\mathrm{C}}$ & $214(1.8)$ & $95(44.4)$ & $1.26(1.00 ; 1.59)$ & $1.21(0.91 ; 1.61)$ \\
\hline Endocrine/ nutritional/ metabolic diseases ${ }^{c}$ & $779(6.6)$ & $358(46.0)$ & $1.34(1.13 ; 1.58)$ & $1.40(1.12 ; 1.74)$ \\
\hline Diseases of the nervous system and the sense organs ${ }^{c}$ & $2,542(21.4)$ & $1,212(47.7)$ & $1.32(1.15 ; 1.53)$ & $1.38(1.13 ; 1.70)$ \\
\hline Epilepsy $^{\mathrm{d}}$ & $429(3.6)$ & $214(49.9)$ & $1.40(1.17 ; 1.68)$ & $1.41(1.11 ; 1.78)$ \\
\hline Brain injury $^{\mathrm{d}}$ & $3,482(29.4)$ & $1,709(49.1)$ & $1.41(1.22 ; 1.63)$ & $1.46(1.19 ; 1.79)$ \\
\hline Diseases of the circulatory system ${ }^{c}$ & $58(0.5)$ & $24(41.4)$ & $1.19(0.78 ; 1.82)$ & $1.40(0.90 ; 2.19)$ \\
\hline Diseases of the respiratory system ${ }^{c}$ & $3,550(29.9)$ & $1,711(31.1)$ & $1.32(1.14 ; 1.52)$ & $1.36(1.12 ; 1.66)$ \\
\hline Asthma $^{\mathrm{d}}$ & $781(6.6)$ & $397(50.8)$ & $1.39(1.18 ; 1.63)$ & $1.46(1.17 ; 1.81)$ \\
\hline Diseases of the digestive system $^{c}$ & $2,737(23.1)$ & $1,267(46.3)$ & $1.32(1.14 ; 1.52)$ & $1.34(1.10 ; 1.64)$ \\
\hline Diseases of the skin and subcutaneous tissue ${ }^{c}$ & $1,414(11.9)$ & $668(47.2)$ & $1.35(1.16 ; 1.57)$ & $1.39(1.13 ; 1.73)$ \\
\hline Atopic disorders $^{\mathrm{d}}$ & $1,062(9.0)$ & $534(50.3)$ & $1.34(1.14 ; 1.57)$ & $1.44(1.16 ; 1.78)$ \\
\hline Diseases of the musculoskeletal system and connective tissue ${ }^{c}$ & $2,699(22.8)$ & $1,263(46.8)$ & $1.35(1.17 ; 1.56)$ & $1.35(1.10 ; 1.65)$ \\
\hline Genitourinary diseases ${ }^{c}$ & $1,848(15.6)$ & $827(44.8)$ & $1.29(1.11 ; 1.50)$ & $1.27(1.03 ; 1.56)$ \\
\hline Complications of pregnancy, childbirth and the puerperium ${ }^{\mathrm{c}}$ & $1,127(9.5)$ & $483(42.9)$ & $1.11(0.94 ; 1.31)$ & $1.14(0.92 ; 1.43)$ \\
\hline Certain conditions originating in the perinatal period ${ }^{c}$ & $1,113(9.4)$ & $523(47.0)$ & $1.24(1.06 ; 1.45)$ & $1.30(1.05 ; 1.60)$ \\
\hline Congenital malformations and chromosomal abnormalities ${ }^{c}$ & $1,095(9.2)$ & $497(45.4)$ & $1.27(1.09 ; 1.49)$ & $1.29(1.04 ; 1.60)$ \\
\hline Autoimmune disorders ${ }^{\mathrm{d}}$ & $332(2.8)$ & $158(47.6)$ & $1.40(1.14 ; 1.71)$ & $1.41(1.10 ; 1.82)$ \\
\hline \multicolumn{5}{|l|}{ Charlsons comorbidity index } \\
\hline 0 & $10,638(89.7)$ & $4,890(46.0)$ & 1.0 (ref.) & 1.0 (ref.) \\
\hline 1 & $946(8.0)$ & $486(51.4)$ & $1.22(1.11 ; 1.34)$ & $1.20(1.09 ; 1.32)$ \\
\hline 2 & $192(1.6)$ & $99(51.6)$ & $1.25(1.03 ; 1.53)$ & $1.23(1.01 ; 1.51)$ \\
\hline $3+$ & $80(0.7)$ & $31(38.8)$ & $0.88(0.62 ; 1.25)$ & $0.79(0.54 ; 1.15)$ \\
\hline
\end{tabular}

Abbreviations: HRR=Hazard rate ratio; 95\%-CI=95\% Confidence Interval; Bold numbers represent significant results even after adjustment for multiple testing.

a The crude analyses are adjusted for age at diagnosis, gender and indexyear.

b The adjusted analyses are adjusted for age at diagnosis, gender, indexyear, in- or outpatient status at incident schizophrenia diagnosis, length of baseline hospitalization,

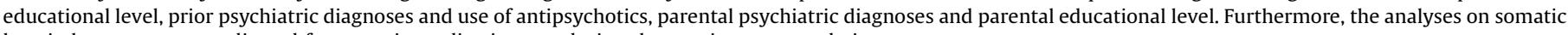
hospital contacts were adjusted for somatic medication use during the previous year and vice versa.

c The overall ICD classes were adjusted for multiple testing, i.e. $\mathrm{p}=0.05 / 15=0.003$.

d The specific diseases were ajusted for multiple testing, i.e. $p=0.05 / 6=0.008$.

e Refers to any redeemed medications during the year prior to the first-time schizophrenia diagnosis.

Table 3

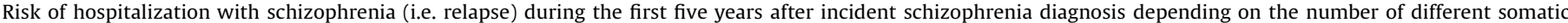
hospital contacts and the time since the last somatic hospital contact.

\begin{tabular}{|c|c|c|c|c|}
\hline & \multicolumn{4}{|c|}{ Any somatic hospital contact } \\
\hline & $\mathrm{N}(\%)$ & Relapse N (\%) & Crude HRR $^{\mathrm{a}}(95 \%-\mathrm{CI})$ & Adjusted HRR ${ }^{\mathrm{b}}(95 \%-\mathrm{CI})$ \\
\hline Eligible individuals & $11,856(100)$ & $5,506(46.4)$ & & \\
\hline No somatic hospital contact & $544(4.6)$ & $220(40.4)$ & 1.00 (ref.) & 1.00 (ref.) \\
\hline Any somatic hospital contact & $11,312(95.4)$ & $5,286(46.7)$ & $1.22(1.06 ; 1.39)$ & $1.30(1.07 ; 1.59)$ \\
\hline \multicolumn{5}{|c|}{ No. different somatic hospital contacts ${ }^{\mathrm{a}}$ : } \\
\hline 1 & $2,629(22.2)$ & $1,228(46.7)$ & $1.18(1.02 ; 1.36)^{c}$ & $1.26(1.03 ; 1.54)^{c}$ \\
\hline $2-3$ & $5,468(46.1)$ & $2,552(46.4)$ & $1.28(1.11 ; 1.46)^{c}$ & $1.33(1.09 ; 1.62)^{\mathrm{c}}$ \\
\hline $4-5$ & $2,431(20.5)$ & $1,105(45.5)$ & $1.29(1.12 ; 1.49){ }^{\mathrm{c}}$ & $1.34(1.09 ; 1.64)^{\mathrm{c}}$ \\
\hline $6+$ & $781(6.6)$ & $401(51.3)$ & $1.50(1.27 ; 1.76)^{c}$ & $1.61(1.29 ; 2.00)^{c}$ \\
\hline \multicolumn{5}{|c|}{ Time since last somatic hospital contact } \\
\hline$<12$ months & $5,039(42.5)$ & $2,386(47.4)$ & $1.27(1.10 ; 1.46)^{\mathrm{C}}$ & $1.36(1.11 ; 1.66)^{\mathrm{c}}$ \\
\hline $1-<2$ years & $1,610(13.6)$ & $651(40.4)$ & $1.04(0.89 ; 1.21)^{\mathrm{c}}$ & $1.12(0.91 ; 1.38)^{\mathrm{C}}$ \\
\hline $2-5$ years & $1,787(15.1)$ & $681(38.1)$ & $0.99(0.85 ; 1.16)^{\mathrm{c}}$ & $1.07(0.87 ; 1.32)^{\mathrm{c}}$ \\
\hline$\geq 5$ years & $1,288(10.9)$ & 437 (33.9) & $0.89(0.75 ; 1.04)^{\mathrm{C}}$ & $1.01(0.81 ; 1.25)^{\mathrm{c}}$ \\
\hline
\end{tabular}

Abbreviations: HRR=Hazard rate ratio; 95\%-CI=95\% Confidence Interval; Bold values represent significant results.

a The crude analyses are adjusted for age at diagnosis, gender and indexyear.

b The adjusted analyses are adjusted for age at diagnosis, gender, indexyear, in- or outpatient status at incident schizophrenia diagnosis, length of baseline hospitalization,

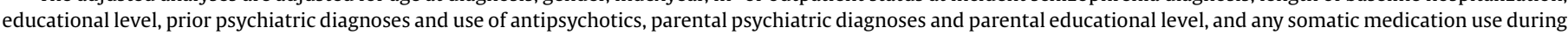
the previous year.

c The risk of relapse increased with a larger number of somatic hospital contacts $(\mathrm{p}<0.005)$, as tested with the Wald's test.

schizophrenia diagnosis showed no significant interaction $(p=0.39)$. Second, we found that a larger amount of somatic hospital contacts during the year prior to first-time schizophrenia diagnosis was associated with increased 5-year-risks for hospitalization with schizophrenia (p<0.005) (Fig. 2 \& Supplementary Table 4 ), with 6 or more prior somatic hospital contacts during the year prior increasing the risk by a HRR of 1.66 (95\%$\mathrm{CI}=1.50 ; 1.84)$. Third, gender interaction analyses did not show a 


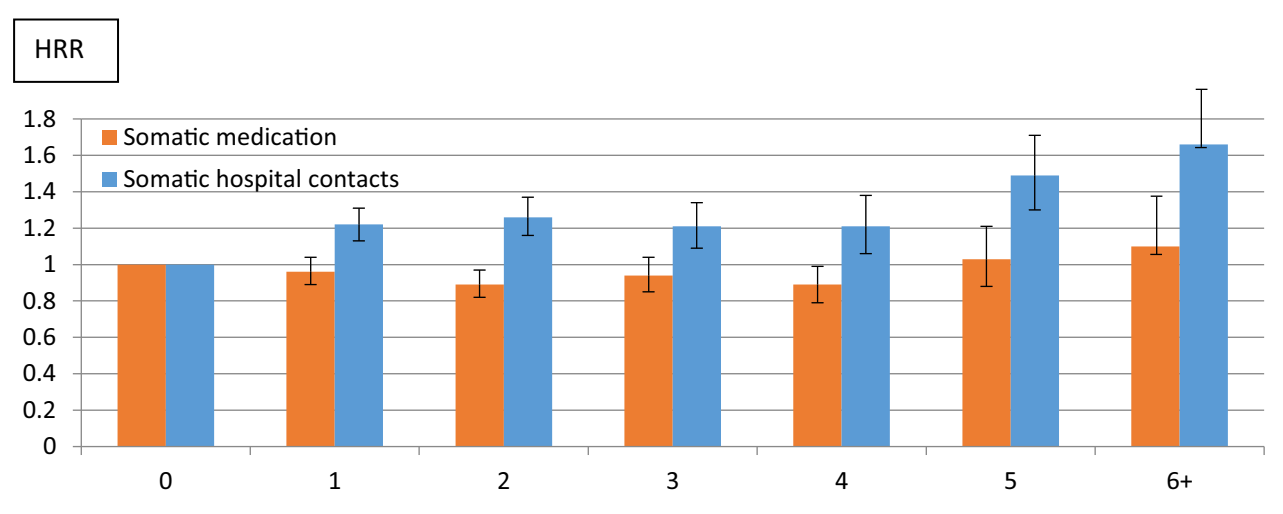

Amount of prescriptions or hospitalizations

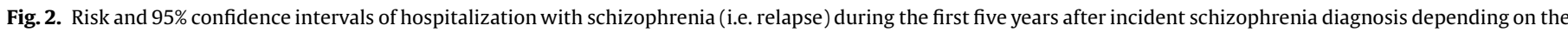
number of different somatic hospital contacts (blue bars) and redeemed somatic medications (red bars) during the year before the incident schizophrenia diagnosis.

significant interaction in the analyses on previous somatic hospital contacts $(p=0.16)$ nor previous somatic medication use $(p=0.06)$.

\section{Discussion}

This nationwide study represents the largest investigation of the association between the entire range of somatic diseases prior to first-time schizophrenia diagnosis and the risk of schizophrenia relapse during the first five years. We included 11,856 patients born after 1977 with a first-time schizophrenia diagnosis between 1996 and 2015 and followed the cohort for 39,033 person-years. Somatic hospital contacts within the year prior to the first-time schizophrenia diagnosis were associated with a $36 \%$ increased risk of schizophrenia relapse, with the risk increasing with the number of somatic diseases. Hence, our findings indicate that severe somatic diseases within the year prior to the first-time schizophrenia diagnosis may negatively affect early schizophrenia treatment course, and that treatment of the somatic disease (as indicated by redeemed prescriptions) may improve treatment course. The latter may also indicate better compliance and help-seeking behavior. We did not find any specific somatic disease(s) to be associated with greater relapse risk and hence no evidence to support that inflammatory diseases were associated with a greater relapse risk.

\subsection{Association between somatic diseases and the early treatment course of schizophrenia}

First, our finding that somatic diseases leading to hospital contacts increased schizophrenia relapse risks indicate that severe somatic diseases negatively affect the early treatment course of schizophrenia. To date, a number of studies have focused on the association between somatic diseases and subsequent development of schizophrenia $[1,3,4]$ respectively the higher mortality risk among patients with schizophrenia [10,11]. Epidemiologic evidence suggests that somatic diseases occur more frequently among patients with schizophrenia compared to non-psychiatric patients [1] and that somatic comorbidity is attributable for more than half of the reduced expected life expectancy [10]. Despite these important associations, to date, only two clinical studies associated higher BMI with an increased readmission risk among 224 psychiatric patients [8] respectively somatic conditions with more time spent depressed and longer duration of illness among 482 patients with bipolar disorder [9]. An observational study found an association between chronic somatic diseases and an increased 30 day re-hospitalization risk among 3563 patients with schizophrenia [22]. One register-based study has shown that prescriptions for non-steroidal anti-inflammatory drugs (NSAIDs) during the early schizophrenia treatment course, indicating the presence of painful somatic conditions, were associated with increased schizophrenia relapse risks [23]. Finally, another register-based study found that patients with higher inflammatory markers had a higher mortality rate, but that the risk for relapse was not affected [24]. However, despite studies indicating that somatic diseases among patients with schizophrenia may be undertreated [25], the present study is the first to investigate the overall impact of life-time somatic diseases on treatment course, indicating that previous somatic comorbidity indeed may affect the early schizophrenia treatment course.

Our findings that somatic diseases in schizophrenia predict increased risks of schizophrenia relapse could point to several underlying explanations. First, it is possible that serious co-morbid somatic diseases add complexity to the psychopharmacological treatment. Secondly, the threshold to become readmitted with schizophrenia could be influenced by having a potentially life threatening co-morbid disease. Third, a patient's compliance with respect to antipsychotic medication could be influenced by the number of co-morbid somatic diseases. Fourth, having a severe somatic disease could also cause somatic anxiety which could affect the psychological well-being as well as help-seeking behavior.

Secondly, we found no associations between redeemed prescriptions for somatic medications and an increased risk for schizophrenia re-hospitalization. Rather, our findings indicate a trend that individuals who had redeemed prescriptions for somatic medications during the year before the first-time schizophrenia diagnosis had a slightly reduced risk for relapse. This indicates that treatment of a somatic disease may improve the treatment course of the mental disorder, in this case schizophrenia. These findings may also indicate that the patients had more help-seeking behavior and better compliance as they were able to see their general practitioner and redeem prescriptions at the pharmacy. Nonetheless, it has been shown in previous studies that sufficient treatment of somatic diseases can significantly reduce the mortality risk [26,27]. Taken together, these results emphasize that diagnosing and treating somatic diseases among individuals with schizophrenia seem to improve the life and the early clinical course of these individuals. 


\subsection{Strengths and limitations}

The strengths of the present study are the nationwide setting and the well-validated Danish registers [12-16]. Importantly, the validity of the schizophrenia diagnoses in the registers is high [17]. In addition, for the present study, we only included individuals with life-long information on all contacts to somatic and psychiatric hospital settings, yielding life-long information for both exposure and outcomes. Finally, the prescription register gives nationwide information on all redeemed prescriptions.

Limitations include that we had a relatively young study population aged up to a maximum of 39 years to capture life time disease burden. Second, we had no information on whether individuals visited their general practitioner without redeeming prescriptions. It has been shown that general practitioner visits increase prior to the first schizophrenia diagnosis [28]. Third, we had only information whether patients redeemed prescriptions but no information on actual compliance, both regarding somatic and psychotropic medication. Fourth, we had no information on the severity of the somatic disease nor the schizophrenia disorder.

\subsection{Conclusion}

The present study found that severe somatic diseases may increase the risk for schizophrenia relapse among patients with a first-time schizophrenia diagnosis, whereas patients who redeemed prescriptions for somatic diseases showed a trend towards slightly lower relapse risks. These findings emphasize the need for a good and broad assessment of somatic diseases among patients with schizophrenia and relevant treatment hereof, as this also may improve the treatment course of the schizophrenia disorder

\section{Appendix A. Supplementary data}

Supplementary material related to this article can be found, in the online version, at doi:https://doi.org/10.1016/j.eurpsy.2019.03.006.

\section{References}

[1] Sorensen HJ, Nielsen PR, Benros ME, Pedersen CB, Mortensen PB. Somatic diseases and conditions before the first diagnosis of schizophrenia: a nationwide population-based cohort study in more than 900000 individuals. Schizophr Bull 2015;41(2):513-21, doi:http://dx.doi.org/10.1093/schbul/ sbu110.

[2] Orlovska S, Pedersen MS, Benros ME, Mortensen PB, Agerbo E, Nordentoft M. Head injury as risk factor for psychiatric disorders: a nationwide registerbased follow-up study of 113,906 persons with head injury. Am J Psychiatry 2014;171(4):463-9, doi:http://dx.doi.org/10.1176/appi.ajp.2013.13020190.

[3] Benros ME, Nielsen PR, Nordentoft M, Eaton WW, Dalton SO, Mortensen PB. Autoimmune diseases and severe infections as risk factors for schizophrenia: 30-year population-based register study. Am J Psychiatry 2011;168(12):130310, doi:http://dx.doi.org/10.1176/appi.ajp.2011.11030516.

[4] Nielsen PR, Benros ME, Mortensen PB. Hospital contacts with infection and risk of schizophrenia: a population-based cohort study with linkage of Danish national registers. Schizophr Bull 2014;40(6):1526-32, doi:http://dx.doi.org/ $10.1093 / \mathrm{schbul} / \mathrm{sbt} 200$.

[5] Blomstrom A, Karlsson $H$, Svensson A, Frisell T, Lee BK, Dal $H$, et al. Hospita admission with infection during childhood and risk for psychotic illness-A population-based cohort study. Schizophr Bull 2013, doi:http://dx.doi.org/ $10.1093 /$ schbul/sbt195.

[6] Köhler O, Petersen L, Mors O, Mortensen PB, Yolken RH, Gasse C, et al Infections and exposure to anti-infective agents and the risk of severe mental disorders: a nationwide study. Acta Psychiatr Scand 2017;135(2):97-105, doi: http://dx.doi.org/10.1111/acps.12671.

[7] Pedersen MS, Benros ME, Agerbo E, Borglum AD, Mortensen PB. Schizophrenia in patients with atopic disorders with particular emphasis on asthma: a
Danish population-based study. Schizophr Res 2012;138(1):58-62, doi:http:// dx.doi.org/10.1016/j.schres.2012.02.019.

[8] Manu P, Khan S, Radhakrishnan R, Russ MJ, Kane JM, Correll CU. Body mass index identified as an independent predictor of psychiatric readmission. J Clin Psychiatry 2014;75(6):e573-7, doi:http://dx.doi.org/10.4088/JCP.13m08795.

[9] Sylvia LG, Shelton RC, Kemp DE, Bernstein EE, Friedman ES, Brody BD, et al. Medical burden in bipolar disorder: findings from the clinical and health outcomes initiative in comparative effectiveness for bipolar disorder study (bipolar CHOICE). Bipolar Disord 2015;17(2):212-23, doi:http://dx.doi.org/ $10.1111 /$ bdi.12243.

[10] Laursen TM, Munk-Olsen T, Agerbo E, Gasse C, Mortensen PB. Somatic hospital contacts, invasive cardiac procedures, and mortality from heart disease in patients with severe mental disorder. Arch Gen Psychiatry 2009;66(7):713-20, doi:http://dx.doi.org/10.1001/archgenpsychiatry.2009.61.

[11] Nordentoft M, Wahlbeck K, Hällgren J, Westman J, Osby U, Alinaghizadeh H, et al. Excess mortality, causes of death and life expectancy in 270,770 patients with recent onset of mental disorders in Denmark, Finland and Sweden. PLoS One 2013;8(1)e55176, doi:http://dx.doi.org/10.1371/journal.pone.0055176.

[12] Mors O, Perto GP, Mortensen PB. The Danish psychiatric central research register. Scand J Public Health 2011;39(7 Suppl):54-7, doi:http://dx.doi.org/ 10.1177/1403494810395825. https://doi.org/10.1177/1403494810395825.

[13] Lynge E, Sandegaard JL, Rebolj M. The Danish national patient register. Scand J Public Health 2011;39(7 Suppl):30-3, doi:http://dx.doi.org/10.1177/ 1403494811401482. https://doi.org/10.1177/1403494811401482.

[14] Pedersen CB. The Danish civil registration system. Scand J Public Health 2011;39(7 Suppl):22-5, doi:http://dx.doi.org/10.1177/1403494810387965. https://doi.org/10.1177/1403494810387965.

[15] Kildemoes HW, Sorensen HT, Hallas J. The Danish national prescription registry. Scand J Public Health 2011;39(7 Suppl):38-41, doi:http://dx.doi.org/ 10.1177/1403494810394717. https://doi.org/10.1177/1403494810394717.

[16] Jensen VM, Rasmussen AW. Danish education registers. Scand J Public Health 2011;39(7 Suppl):91-4, doi:http://dx.doi.org/10.1177/1403494810394715. https://doi.org/10.1177/1403494810394715.

[17] Uggerby P, Ostergaard SD, Roge R, Correll CU, Nielsen J. The validity of the schizophrenia diagnosis in the Danish psychiatric central research register is good. Dan Med J 2013;60(2)A4578 doi: A4578 [pii].

[18] Olivares JM, Sermon J, Hemels M, Schreiner A. Definitions and drivers of relapse in patients with schizophrenia: a systematic literature review. Ann Gen Psychiatry 2013;12(1), doi:http://dx.doi.org/10.1186/1744-859X-12-32 32-859X-12-32.

[19] Charlson ME, Pompei P, Ales KL, MacKenzie CR. A new method of classifying prognostic comorbidity in longitudinal studies: development and validation. J Chronic Dis 1987;40(5):373-83.

[20] Thygesen SK, Christiansen CF, Christensen S, Lash TL, Sorensen HT. The predictive value of ICD-10 diagnostic coding used to assess charlson comorbidity index conditions in the population-based Danish national registry of patients. BMC Med Res Methodol 2011;11:, doi:http://dx.doi.org/ 10.1186/1471-2288-11-83. . 83-2288-11-83 https://doi.org/10.1186/14712288-11-83.

[21] Nuttall M, van der Meulen J, Emberton M. Charlson scores based on ICD-10 administrative data were valid in assessing comorbidity in patients undergoing urological cancer surgery. J Clin Epidemiol 2006;59(3):265-73, doi:http://dx.doi.org/10.1016/j.jclinepi.2005.07.015.

[22] Boaz TL, Becker MA, Andel R, Van Dorn RA, Choi J, Sikirica M. Risk factors for early readmission to acute care for persons with schizophrenia taking antipsychotic medications. Psychiatr Serv 2013;64(12):1225-9.

[23] Kohler O, Petersen L, Benros ME, Mors O, Gasse C. Concomitant NSAID use during antipsychotic treatment and risk of 2-year relapse - a population-based study of 16,253 incident patients with schizophrenia. Expert Opin Pharmacother 2016;1-8, doi:http://dx.doi.org/10.1517/ 14656566.2016.1168400.

[24] Horsdal HT, Köhler-Forsberg O, Benros ME, Gasse C. C-reactive protein and white blood cell levels in schizophrenia, bipolar disorders and depression associations with mortality and psychiatric outcomes: a population-based study. Eur Psychiatry 2017;44:164-72.

[25] Laursen TM, Munk-Olsen T, Gasse C. Chronic somatic comorbidity and excess mortality due to natural causes in persons with schizophrenia or bipolar affective disorder. PLoS One 2011;6(9)e24597, doi:http://dx.doi.org/10.1371/ journal.pone.0024597.

[26] Kugathasan P, Horsdal HT, Aagaard J, Jensen SE, Laursen TM, Nielsen RE. Association of secondary preventive cardiovascular treatment after myocardial infarction with mortality among patients with schizophrenia. JAMA Psychiatry 2018;75(12):1234-40.

[27] Laursen TM, Mortensen PB, MacCabe JH Cohen D, Gasse C. Cardiovascular drug use and mortality in patients with schizophrenia or bipolar disorder: a Danish population-based study. Psychol Med 2014;44(Jun (8)):1625-37.

[28] Nørgaard H, Søndergaard Pedersen H, Fenger-Grøn M, Mors O, Nordentoft M, Vestergaard M, et al. Increased use of primary care during 6 years of prodromal schizophrenia. Acta Psychiatr Scand 2016;134(3):225-33. 